\title{
Assessing Available Transfer Capacity on a Realistic European Network: Impact of Assumptions on Wind Power Generation
}

\author{
Vincent Rious, Julio Usaola, Marcelo Saguan, Jean-Michel Glachant, Philippe Dessante
}

\begin{abstract}
This paper aims at assessing the impact of massive wind power penetration on the calculation of Available Transfer Capacity (ATC) for the interconnections between European countries. Calculations are made for the ATC between France and Belgium and are realized on a realistic European Electricity Network. We find that the German wind power production make this ATC vary depending on the total wind power production and its geographical distribution in Germany. Wind power production and the nodes involved in cross-border exchange must then be forecast precisely so that the cross-border exchange can be maximal without breaching network security.
\end{abstract}

\section{INTRODUCTION}

$\mathrm{L}$ imited transmission interconnection capacities are still

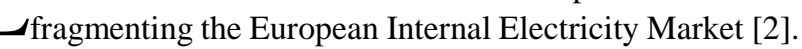
Increasing available interconnection capacities between countries is crucial to integrate markets and to benefit from more competition and more efficiency [13][14]. However the actual cross-border congestion management methods may prevent from using all physical interconnection capacity. At the same time strong incentives on the development of wind power are being given and large penetration of this intermittent energy are now more the rule than the exception [11]. High penetration of wind power may worsen the fragmentation of the European Electricity Markets. Indeed it is required to reduce available transmission capacities to keep the power system safe despite the uncertainty about wind power.

The use of suboptimal transmission capacity allocation methods explains partially why the European Electricity Market is still not integrated ${ }^{1}$. After a heated debate about the type of transmission pricing and capacity allocation, the actual applied methods differ from methods theoretically optimal (e.g. nodal pricing, etc.). Actual methods applied in Europe go from explicit and non coordinated transmission capacity auctions (separated from the energy markets) to implicit partially coordinated transmission capacity auctions (integrated with the energy markets). In both methods

Manuscript received April 19, 2008. This work was supported in part by the French Regulatory Commission (CRE) under the €nergy Project II and in part by the Region of Ile de France under the R2DS program.

V. Rious and P. Dessante are with Supelec, Gif-sur-Yvette, 91190 France (corresponding author: V. Rious; phone: +33 (0)169851531; fax: +33 (0)169851 539; e-mail: vincent.rious@supelec.fr).

M. Saguan and J.-M. Glachant are with University Paris-Sud XI, Fontenay-aux-Roses, 92260 France.

J. Usaola is with University of Carlos III de Madrid, Leganés 28911, Spain
European countries grid are still represented by only one zone (one node) and cross-border interconnection capacities are computed following the concept of Available Transfer Capacity (hereafter ATC, [6]).

The concept of ATC implies that the physical Kirchhoff laws that describe the power flow are partially ignored when the market for power or for transmission capacity is cleared day-ahead. It is then assumed that the power flows through interconnections behave as water or any material flow. For this approximation to be made possible, ATC calculation requires different assumptions on where power is generated and consumed without day-ahead exchanges, on where power exchanges are generated and consumed. Such assumptions could be aggregated in two items: the choice of exchange nodes and the choice of the "base case", i.e. the supposed load flow in the network to start ATC calculations. Since the intermittency of wind power can greatly modify the location of generation on the network, it may have a critical impact on the calculation of Available Transfer Capacity.

A commonly known example is the case of wind power in Germany that may impact the transmission capacity available for the cross-border exchange in the central western European area, that is to say for exchange between France, Belgium, the Netherlands, and Germany [10]. Indeed, the networks of these countries are highly meshed and injections and withdrawals in one of these areas may greatly impact power flows in the other areas and cross-border flows. This is not a problem if injections and withdrawals can be predicted, since flows can then also be predicted ${ }^{2}$. However, when there is uncertainty on injections, as for wind power for instance, there is also uncertainty on base case power flows, and then on transmission capacity available for cross-border exchanges. Indeed ATC has to be reduced to ensure that load flows are feasible even in the worst possible case.

The aim of this paper is twofold. Firstly this paper illustrates and analyzes how wind power generation may impact ATC calculation. Secondly this paper assesses the impact of wind power penetration on the ATC calculation over a realistic European Network, considering the impact of German wind power on the calculation of ATC for exchange

\footnotetext{
${ }^{1}$ The lack of cross-border investments has been presented as another important reason [2].

${ }^{2}$ In this paper, we will assume that the TSOs exchange enough data to calculate the best possible values of ATC. This is a strong assumption that may not be true in reality, as suggested by the ETSO report [8]. The unique issue we consider in this paper is then only related to the important variability of wind power and its impact on the calculation of ATC.
} 
between France and Belgium.

This paper is organized as follow. In section II we explain why we need to define ATC to implement congestion management methods in Europe and how ATC are computed showing a three-node example. In section III we analyze the impact of wind power on ATC for the case of Europe. Section IV concludes and comments further researches.

\section{WHY WE NEED ATC AND HOW THEY ARE COMPUTED}

The variety of cross-border congestion management schemes is quite wide in Europe. But one common denominator is the way the transmission capacity is calculated relying on the concept of ATC. ${ }^{3}$

This section presents the diversity of transmission pricing in Europe and why the European electricity market relies on ATC to define cross-border transmission capacity. The second part of this section presents how TSOs generally calculates ATC in Europe. Finally, the third part of this section shows how different assumptions (exchange node and base case) may modify ATC calculations.

\section{A. ATC and Electricity Transmission Pricing in Europe}

Many different congestion management schemes are actually applied in Europe [9]. Two choices of design characterized each of these schemes: $1^{\circ}$ Cross-border transmission auctions' coordination and $2^{\circ}$ Bundling transmission \& energy (commodity) auctions.

The first point of design for transmission capacity auction deals with how TSOs coordinate to calculate and allocate the cross-border capacity. The transmission capacity auction can be then "non-coordinated" (i.e. each border is treated independently of others) or "coordinated" where TSOs coordinate and communicate each other data to increase the accuracy of calculation of transmission capacity and make cross-border coordinated auctions. The second point considers whether the chosen auction for congestion management is "explicit" or "implicit". If it is explicit, energy and transmission capacity are separately sold. If the auction is implicit, energy and transmission capacity are sold bundled, which ensures an optimal use of the auctioned transmission capacity [16][5]. With these two points, the design of actual transmission capacity auctions applied in Europe span from explicit and non coordinated transmission capacity auctions (e.g. France-Germany) whose efficiency can be doubtful to implicit partially coordinated transmission capacity auctions whose efficiency is recognized (e.g. France-Belgium-Netherlands).

No matter the congestion management schemes used in each border in Europe all of them share the same way for calculating cross-border transmission capacity. Indeed the definition of cross-border transmission capacity then relies on the concept of ATC. Relying on ATC to define cross-border

\footnotetext{
${ }^{3}$ The application of the principle of subsidiarity explains why the design of transmission pricing in Europe needs to rely on this concept.
}

transmission capacity is a compromise to manage cross-border congestion as efficiently as possible while considering each country as a hub. The next subsection shows how ATC are calculated.

\section{B. Computing ATC}

In this paper, we will study ATC computed for day-ahead transactions. ATC will then be computed on a country-wide basis, since, to the exception of Norway and Italy that have values of ATC for internal constraints, ATC are all country-wide across Europe [9].

To define ATC, one first needs to recall the definition of the Total Transfer Capacity (TTC) and of the Net Transfer Capacity (NTC) [6]. The TTC is the maximum exchange program between two areas compatible with operational security standards applicable at each system if future network conditions, generation and load patterns were perfectly known in advance. The NTC is the maximum exchange program between two areas compatible with security standards applicable in both areas and taking into account the technical uncertainties on future network conditions mostly year ahead. The NTC is then calculated from the TTC subtracting a Transmission Reliability Margin (TRM) (see equation 1).

NTC $=$ TTC-TRM

The ATC is the cross-border additional exchanges that remain possible, after each phase of the allocation procedure of the transmission capacity (see equation 2 ).

ATC=NTC-Already allocate capacity

The possibilities for import/export transactions in a meshed interconnected network (such as the European ones) between two countries (i.e. Available Transfer Capacity) depend on all realized transactions -also between other than the two considered countries -due to the so called parallel flows which are the direct consequence of physical laws of electrical flows in the interconnected networks. Thus the maximum possible use of the capacity between two given countries depends to some extent on all local as well as on all distant transactions, because they rely on the European production plans and on the consumer loads $[7]^{4}$.

This statement has three consequences for the calculation of ATC. $1^{\circ}$ The "base case" defining injections and withdrawals before the day-ahead exchanges taking place must be known with accuracy, node by node. Only then the ATC can be known with accuracy too. $2^{\circ}$ While calculating the ATC for an exchange between two countries, the effect of other cross border exchange between other than the two considered countries must also be included in this computation. $3^{\circ}$ The possible cross-border transfer between two countries is dependent on the choice of the node (or set of nodes) in each country involved in this exchange. Different couples of nodes have indeed different influences on power flows. Therefore, to define an ATC between two set of nodes, it is needed to choose the set of nodes in the exporting country where to

\footnotetext{
${ }^{4}$ See also http://www.rte-france.com/htm/fr/offre/offre inter capa.jsp, seen the $9^{\text {th }}$ of April 2008 .
} 
inject power and the set of node in the importing country where to withdraw power.

These three elements constitute the skeleton of the process for computing ATC can be computed respecting the following process (see footnote 4 ).

1. Choice of the base case situation and calculation of the base case flows

2. Choice of one set of nodes $\{\mathrm{m}\}$ in the exporting country and one set of nodes $\{\mathrm{n}\}$ in the importing country, nodes that participate in the cross-border exchange

3. Calculation of the real available physical transmission capacity for each line (other things equal)

4. Calculation of the physical transmission capacity available for the considered cross-border exchange on each line (other things equal)

5. Calculation of the maximum commercial exchange that can additionnally flow on each line (other things equal)

6. Calculation of the ATC

Fig. 1 Schema of ATC assessment process

1. A base case of generation and load node by node allows to calculate base case power flows on all transmission lines, including and especially on interconnection ones. Generation and consumption used to define the base case are the results of power transactions before the day-ahead ones (week-ahead, month-ahead, year-ahead). The TSO knows only the difference between total generation and total load on its control area. He must anticipate the nodal sharing of total generation and load to forecast the base case power flows. This anticipation of nodal data from zonal data is a source of uncertainty. The base case power flow on transmission line between node $\mathrm{i}$ and $\mathrm{j}$ is $\mathrm{P}_{\mathrm{ij}}{ }^{\circ}$. Only a subset of possible congested lines (or other power system facility) is monitored. For simplicity we concentrate in tielines, i.e. cross-border lines that connect national systems. ${ }^{5}$

2. The TSO must also anticipate the nodes that will participate in the cross-border exchange. There exist different way to represent cross-border exchange: choice of two representative nodes; pro-rata redispatching; economical redispatching, etc. [7]. For simplicity we continue the explanation supposing that one couple of nodes (one node in the exporting zone and one node in the importing zone) is chosen by the TSO for ATC calculation. We note $\mathrm{m}$ the exporting node and $\mathrm{n}$ the importing node. The choice of these nodes has an impact on the available transmission capacity, as the influence on a tieline may be a flow in the same direction as the base case flow or a counter flow regarding the base case

\footnotetext{
${ }^{5}$ ATC computation includes also lines (e.g. internal lines) that may be congested when cross-border exchange increases.
}

flow.

3. The coefficient PTDF $F_{i j, m n}$ defines the additional flow on tieline ij for an exchange between two nodes $m$ and $n$. These coefficients can be calculated with different assumptions regarding the network physical laws and security criteria. They can be calculated using an AC or a DC load flow. Under the assumptions of DC load flow, the physical laws of electricity are simplified and linearized [17]. But DC PTDF coefficients still remain good approximations of AC PTDF [4]. The PTDF coefficients can also be calculated with or without N-1 security criterion. An N-1 or more generally N-k rule is a security rule designed such that the power system keeps safe at any moment even after a hypothetical random event on a nominal situation that leads to the loss of $\mathrm{k}$ elements of the network [17].

If the PTDF $\mathrm{ij}_{\mathrm{ij}, \mathrm{mn}}$ has the same sign as the base case flow on line $\mathrm{ij}$, the additional flow is in the same direction as the base case power flow. If $\mathrm{PTDF}_{\mathrm{ij}, \mathrm{mn}}$ has a sign different from the base case flow on line $\mathrm{ij}$, the additional flow is a counterflow with regard to the base case flow. More generally, the available capacity on each tieline $\mathrm{ij}$ is then given by the equation 3.

$$
\mathrm{P}_{\mathrm{ij}}{ }^{\mathrm{av}-\mathrm{real}}=\left(\mathrm{P}_{\mathrm{ij}}{ }^{\max }-\operatorname{sign}\left(\mathrm{PTDF}_{\mathrm{ij}, \mathrm{mn}}\right) \mathrm{P}_{\mathrm{ij}}{ }^{\circ}\right)
$$

4. When ATC are calculated for more than one border one supplementary assumption should to be made. In fact available capacity in each possible congested tieline has to be "shared" between the different borders. A common assumption used in Europe is that the available capacity of each tieline $\mathrm{ij}$ is equally shared between the $\mathrm{k}$ different cross-border exchanges that influence the tieline ij (see footnote 4). Then, the defined ATC are then simultaneously feasible.

$$
\mathrm{P}_{\mathrm{ij}}{ }^{\mathrm{av}}=\left(\mathrm{P}_{\mathrm{ij}}{ }^{\max }-\operatorname{sign}\left(\mathrm{PTDF}_{\mathrm{ij}, \mathrm{mn}}\right) \mathrm{P}_{\mathrm{ij}}{ }^{\mathrm{o}}\right) / \mathrm{k}
$$

For instance, a tieline between France and Belgium is influenced by exchanges between France and Belgium, France and Germany, France and Switzerland, and for a little share by France and Italy. ${ }^{6}$ In this case, the available capacity on each tieline ij should be divided by 4 .

5. All things equal, an exchange between two nodes $m$ and $n$ (where $\mathrm{m}$ and $\mathrm{n}$ are in different countries) can then occur up to result of equation 4.

$\mathrm{Ex}_{\mathrm{mn}, \mathrm{ij}}{ }_{\max }=\left(\mathrm{P}_{\mathrm{ij}}^{\max }-\operatorname{sign}\left(\mathrm{PTDF}_{\mathrm{ij}, \mathrm{mn}}\right) \mathrm{P}_{\mathrm{ij}}^{\circ}\right) /\left(\mathrm{k} \cdot \mid \mathrm{PTDF}_{\mathrm{ij}, \mathrm{mn}}{ }^{\prime}\right)(5)$

6. The $\mathrm{ATC}_{\mathrm{mn}}$ for an exchange between two nodes $\mathrm{m}$ and $\mathrm{n}$ (each one in a different country) is the minimum value of $\mathrm{Ex}_{\mathrm{mn}, \mathrm{ij}}{ }^{\mathrm{max}}$ for the different tielines $\mathrm{ij}$, that is to say

$\mathrm{ATC}_{\mathrm{mn}}=\min \left\{\left(\mathrm{P}_{\mathrm{ij}}^{\mathrm{max}}-\mathrm{P}_{\mathrm{ij}}{ }^{\circ}\right) /\left(\mathrm{k} \cdot \mathrm{PTDF}_{\mathrm{ij}, \mathrm{mn}}\right), \mathrm{ij}\right\}$

To conclude this sub-section it is important to note that two elements are relevant for the calculation of ATC. First the choice of the node where to inject power in the exporting country and the node where to withdraw power in the importing country changes the resulting power flows related to the considered cross-border transfer. Second changing the

\footnotetext{
${ }^{6}$ This step of the process makes quite unreal that a line be really congested when ATC constrain cross-border exchanges.
} 
base case changes the available physical capacity on power lines and so the available transfer capacity. In the next subsection we use a three-node example in order to show the impact of these two elements on the ATC calculations.

\section{Example of the impact of base case and of exchange nodes}

Fig. 2 represents the stylized three-node system. There are two zones/countries, country A with only one node (1) and country B with two nodes (2 and 3 ) and an internal line. Two tielines interconnect zones A and B: line 1-2 and line 1-3. All lines have the same impedance and PTDF are represented in the figure. Line 1-2 has a thermal limit of $100 \mathrm{MW}$ while line 1-3 has much more higher limit (infinite in theory). The "base case" is also represented in the figure; this corresponds to an injection of $100 \mathrm{MW}$ in node 1 and withdrawals of $50 \mathrm{MW}$ in nodes 2 and 3 .

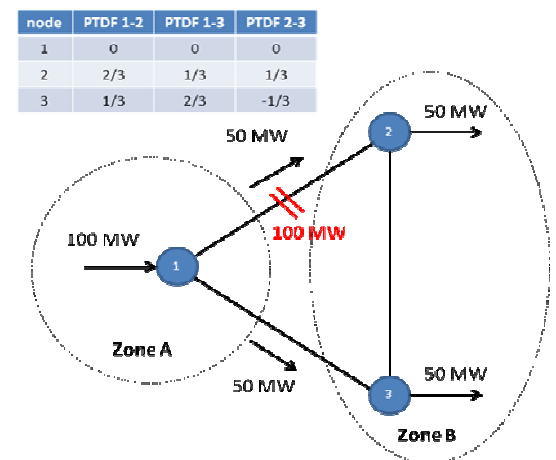

Fig. 2 Stylized 3-nodes system: No Wind base case

Let's study the impact of the choice of nodes of exchange in order to compute ATC between the two zones. Taking the base case we have to increase the exchange between zones until the thermal limit is attained. If we select nodes 1 and 2 to make the exchange we have that thermal limit is attained once supplementary cross-border exchange is $75 \mathrm{MW}$, thus the ATC is equal to $75 \mathrm{MW}$ (50 free MW in line 1-2 divided by PTDF of line 1-2 and node 2). If we select nodes 1 and 3 to make exchange we have an ATC of $150 \mathrm{MW}$. Therefore we can see in this simple example the importance of the choice of nodes. It is important to note that the best choice of nodes should correspond to the actual situation. As at the moment of computing ATC TSOs don't have all the relevant information, the choice of nodes have to correspond to the best estimate of actual situation.

Let's now consider that there are uncertainties in the definition of the base case. This corresponds for instance when there is high penetration of wind power in one of zones and there is uncertainty of wind power generation. Let's suppose that the base case have two possibilities: the first possibility, "no wind base case" is such represented in Fig. 2 and the other possibility, "high wind base case" is represented in Fig. 3. Here, there is an injection of $30 \mathrm{MW}$ of wind power in node 2 and a decrease in generation in node 3 (or increase of consumption). ATC computed with this wind base case are: using nodes 1 and 2, ATC is $90 \mathrm{MW}$ and using nodes 1 and 3, ATC is 180 .



Fig. 3 Stylized 3-nodes system: "Wind Base case"

Table I summarizes ATC calculations with the different assumptions. This table illustrates simply how the choice of nodes and the wind power assumption can impact on the final value of ATC.

TABLE I

ATC FOR DIFFERENT BASE CASES AND EXCHANGE NODES ASSUMPTIONS

\begin{tabular}{c|c|c}
\hline \multicolumn{3}{c}{ Base case } \\
\hline Nodes & No Wind & Wind \\
\hline \hline 1 and 2 & $75 \mathrm{MW}$ & $90 \mathrm{MW}$ \\
\hline 1 and 3 & $150 \mathrm{MW}$ & $180 \mathrm{MW}$ \\
\hline
\end{tabular}

In conclusion, the two main assumptions may impact ATC values: the base case and the choice of exchange nodes. As the goal of this paper is to study the influence of wind power on ATC calculation, we proceed this paper considering only the assumptions of the base case and in particular the modification of the base case by the wind power generation to study these effects in a realistic European Network.

\section{THE EFFECTS OF WIND POWER IN EU ATC}

In this section we assess how the wind power penetration may impact the ATC calculation in Europe, considering the impact of German wind power on the calculation of ATC for exchange between France and Belgium. In order to do so we propose a preliminary evaluation of ATC with simplifying assumptions on the European network published by [18]. ${ }^{7}$

Here we choose only one couple of nodes to realize a cross-border exchange between France and Belgium. ${ }^{8}$ The ATC between France and Belgium with this couple of node

${ }^{7}$ In [18] Zhou and Bialek built a simplified but realistic representation of the European Network, more precisely of the area number 1 of UCTE (without the Balkan country and in the far South West Europe - Bulgaria, Romania) in 2002. There are 1254 nodes and 1918 lines. And for the countries we are interested in, there are 316 nodes in France, 46 in Belgium, and 227 in Germany. And there are 21 cross-border lines between France and the neighboring countries.

${ }^{8}$ In this paper, we assume that only one node participates in each country (respectively an exporting and an importing one) in a cross-border exchange. More generally, several nodes may be involved in a cross-border exchange. But, for a TSO to consider that several nodes participate in a cross-border exchange, he must consider a merit order [3]. 
and without wind power generation is $580 \mathrm{MW}$. We use the base case from [18] for our base case without wind power ${ }^{9}$. In this section, we will then see the modification of base case brought by wind generation.

To do so, we first explain methodology, assumptions and data to assess ATC under different level of wind power production, then we present results of our simulations and finally, we discuss results.

\section{A. Methodology \& Data for wind power production: Probability Density Functions}

Due to the huge difficulty of knowing the production of wind farms throughout Europe for a long enough time, a simulation exercise will be done in order to assess the impact of German wind farms on the France-Belgium ATC. This simulation, based on realistic assumptions and following the stochastic nature of wind energy, will produce reliable results that allow to draw out valid conclusions.

Wind power production can be considered as a random variable with a given Probability Density Function (PDF). The shape of this PDF is given by the distribution of wind speed in a site, which is usually represented by a Weibull distribution, and the relation between wind speed and power, that comes given by the P-v relationship. In the resultant distribution, the possibility of a very short production is usually quite high.

Another feature of wind power production is that the generation of different wind farms throughout a wide area is correlated, because they are due to similar meteorological situations.

In order to simulate German wind power generation, different wind generation scenarios have been made taking into account PDF aggregation and correlation features with Monte Carlo simulation. The method has been the following one. First multivariate normal random numbers with a given correlation have been generated. Then, through an inverse transformation, a set of correlated numbers, uniformly distributed, has been obtained. From them, a new transformation has been made to generate random numbers with the chosen marginal distributions.

To generate the wind power production scenarios, we made the four following assumptions. Firstly, we assume that total installed capacity in Germany is $14000 \mathrm{MW}$. It is the wind power capacity in Germany in 2003 [15], to be coherent with the data from [18] representing the UCTE network in 2002. Secondly, we assume that the wind farms are installed only in the North of Germany, neglecting the $1000 \mathrm{MW}$ wind farms only in the south of Germany. Thirdly, since wind generation is quite equally distributed in the north of Germany, we assume that the remaining $13000 \mathrm{MW}$ of wind power we

\footnotetext{
${ }^{9}$ The base case defined by Zhou and Bialek in [18] if for the peak hour in winter. Load is an estimation of the winter peak load for each node. As for generation, since Zhou and Bialek work without generation cost, they apply in each country a kind of prorata rule on the power generated by each plant regarding national consumption and export or import. The base case power flows are then deduced from nodal generation and load.
}

consider is equally shared between the different sink German nodes, that is to say that the same capacity of power is connected to each load node in Germany. Lastly, the north of Germany has been divided into six zones, and it has been assumed that the production within the same zone has the same level.

The generation of wind power production scenarios has then been made from the following data:

- The installed power in the different points and nodes throughout the German grid.

- A realistic PDF shape of the wind power production level, obtained from measured productions of wind power.

- The correlation coefficients proposed by [12] for the six zones of wind power generation in the north of Germany.

A set of 1000 samples has been obtained, and a Cumulative Densitiy Function has been obtained with the frequencies of occurrences. For a given probability level, a level of overall production can be chosen. This total production can be obtained by different possible combinations of regional productions, which are all equally possible.

Since the addition of wind results in an increase in generation compared to the base case of [18], it is needed to find a way to decrease power generation of power plants other than the wind farms. Here we use the same assumption as [18], i.e. a prorata modification of generation plan. Considering the lack of integration between the European national electricity markets, the dispatchs are mainly run on a national level. Therefore, one can suppose that the power produced by wind generators is compensated by a proportional reduction of the other German generators.

\section{B. Results and discussions}

Before evaluating the effect of wind power on the value of ATC, it is needed to evaluate the feasibility of the base cases with wind power. Since we modify the base case from [18] adding wind power production and decreasing the power production of the other German generators, the power flows are modified and may not be feasible. That is to say that the base case power flows with wind may exceed the maximum capacity of some lines. If such a base case with wind occurred, it should be disregarded from the calculation of ATC. The corresponding scenarios of wind production that lead to infeasible base cases may not be the one with the higher wind power production, since the location of injection is also important in this case, as we assume that the wind power production is not geographically uniform even if correlated.

Fig. 4 shows the variation of the value of ATC between France and Belgium depending on the German wind power production. The $\mathrm{x}$-axis stands for the German wind power production, irrespective of the geographic distribution of this power. The $y$-axis represents the value of ATC in MW. When wind power production is zero, the base case is Zhou and Bialek's ones and the ATC is equal to $580 \mathrm{MW}$ as in [18]. When the base case with wind is not feasible, we set the ATC value at zero. Two main conclusions may be extracted from 
fig. 4: the high proportion of unfeasible base cases and the impact of wind power generation in ATC calculations.

Fig. 4 shows firstly the high proportion of unfeasible base cases with wind is then the numbers of wind scenarios when the ATC is zero. This represents around $85 \%$ of the scenarios in our case. This high proportion of unfeasible base cases with wind is surely due to the rather rough way we modify the generation schedule to keep generation and load in balance. However, without any serious data about generation costs, it is difficult to use another criterion than scaling down proportionally outputs of power plants in a given country. Other studies will then be needed to select more feasible base cases with wind. But in this paper, we will consider only the obtained base cases with wind.

Secondly, on Fig. 4, a trend can be observed, as the ATC between France and Belgium increases with the German wind power production. This is because wind production compensated by the German classic generators schematically creates a counterflow on the lines congested by the cross-border exchanges between France and Belgium. This is similar to the simple example exposed in section II. Beside our observation on this simple example, we observe here on this more realistic case that there is a dispersion of the relation between the German wind power production and the value of ATC. This is because the wind production is not geographically uniform even if there is a correlation of production between the six wind zones that we defined for Germany (see previous subsection). Knowing the exact geographical distribution of wind power in German is then of great importance for an accurate calculation of ATC, between France and Belgium for the present case.

ATC value depending on the total wind power production in Germany

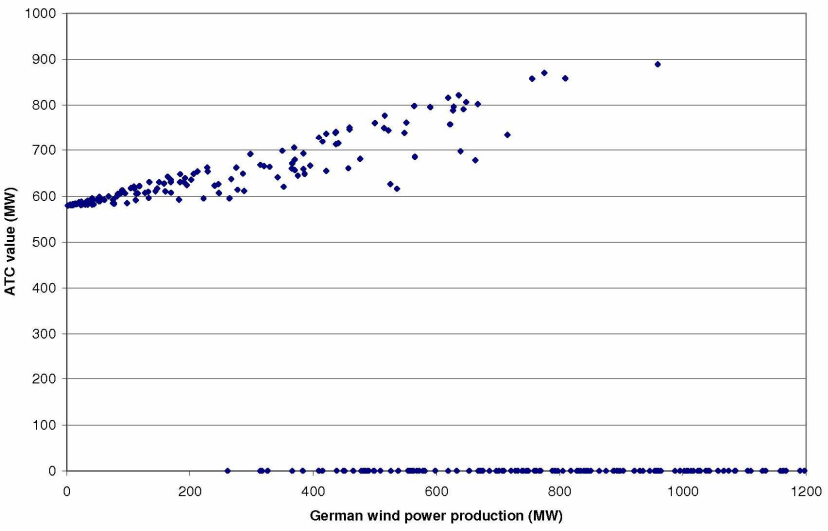

Fig. 4. Values of ATC depending on the German wind power production

\section{CONCLUSIONS AND FUTURE RESEARCHES}

In this paper, we have evaluated the sensitivity of ATC value between France and Belgium to the scenarios of wind power production in Germany. First results indicate that the impact of German wind power production on the value of ATC depends not only on the total German wind power production but also the geographic distribution of production in Germany. The wind power production may have a greater impact on ATC as it is closer to the border between France and Belgium.

Even if we have made several assumptions to proceed these calculations of ATC, it appears clearly here that the anticipation of the TSOs in the Central Western Europe regarding the German wind power production and the node that will be involved in cross-border exchange modify the ATC value. As regard these two elements studied in here, coordination between TSOs is crucial to refine their forecasts.

\section{REFERENCES}

[1] R. Baldick, "Variations of Distribution Factors with Loading", IEEE Transactions on Power Systems 18 (4), 2003, 1316-1323

[2] Direction Générale de la Concurrence (DGComp), DG Competition report on energy sector inquiry, SEC(2006)1724, 2007.

[3] A.Dufour, "Capacity allocation using the flow-based method, Master of Science Thesis, KTH Electrical Engineering Electrical Power System, 2007 www.ee.kth.se/php/modules/publications/reports/2007/XR-EE-ES_20 07 002.pdf

[4] C. Duthaler, M. Emery, G. Andersson and M. Kurzidem, "Analysis of the use of PTDF in the UCTE Transmission Grid", undated, http://www.forum-und-contact.ethz.ch/ duthalec/power-transfer-distri bution-factors-ptdf-duthaler.pdf

[5] A. Ehrenmann and Y. Smeers, "Inefficiencies in European congestion management proposals", Utilities policy 13(2), 2005, 135-152

[6] ETSO, Definition of Transfer Capacity in liberalized Electricity Markets, April 2001, www.etsonet.org

[7] ETSO, Procedures for cross-border transmission capacity assessments, October 2001, www.etsonet.org

[8] ETSO, List of data TSOs need to pursue optimal use of the existing infrastructure, December 2005

[9] ETSO, An Overview of Current Cross-border Congestion Management Methods in Europe, May 2006, www.etso-net.org

[10] ETSO-UCTE, The Electricity Transmission System Operators views on the Integration of Wind Energy in the European Electricity System, $22^{\text {nd }}$ February 2006, www.etso-net.org

[11] EurObserv'ER, "le baromètre éolien, SYSTÈMES SOLAIRES le journal des énergies renouvelables 183", 2008, http://www.energies-renouvelables.org/observ-er/stat_baro/observ/bar o171.pdf

[12] U. Focken, M. Lange, K. Mönnich, H.-P. Waldl, H. G. Beyer and A. Luig, "Short-term prediction of the aggregated power output of wind farmsFa statistical analysis of the reduction of the prediction error by spatial smoothing effects". Journal of Wind Engineering and Industrial Aerodynamics 90, 2002, 231-246,

[13] JM. Glachant and F. Lévêque, "Electricity Internal Market in the European Union: What to do next?", CEEPR Working Paper 2005

[14] JM. Glachant, Y. Perez, V. Pignon and M. Saguan, "Un marché européen de l'électricité ou des marchés dans l'Europe? Regards croisés : économistes et ingénieur", Droit de la régulation et l'économie, Annales de l'UMR, André TUNC, Université Paris I, May 2005

[15] R. Madlener and M. Schneider, "Economic modelling of the diffusion of wind power in Germany: comparison of approaches and policy implications". 3rd European Congress on the "Economics and Management of Energy in Industry" (ECEMEI 2004), 6-9 ${ }^{\text {th }}$ April 2004, Estoril-Lisbon, Portugal.

[16] K. Neuhoff, "Coupling Transmission and Energy Markets Reduces Market Power", Cambridge Working Papers in Economics 2003

[17] RTE, Mémento de sûreté, 2004, available on www.rte-france.com

[18] Q. Zhou and J. Bialek, "Approximate Model of European Interconnected System as a Benchmark System to Study Effects of Cross-Border Trades", IEEE Transactions on Power Systems 20 (2), 2005, 782-788 